\title{
Conversion of Endoscopic Nasobiliary Drainage to Internal Drainage by Means of Endoscopic Scissor Forceps
}

Endoscopic nasobiliary drainage (ENBD) [1] may cause undue stress, such as pharyngeal discomfort, in some patients. We have developed a new method for conversion from external to internal drainage by cutting the external drainage tube with endoscopically employed scissor forceps, which were developed in collaboration by the authors and the Olympus Company (Tokyo, Japan). The forceps can cut a tube with a maximum diameter of 7-F, and are designed so that the ENBD tube can be held at the depressed portion with the blade almost perpendicular to the longitudinal axis of the tube (Figure $\mathbf{1}$ ). The external drainage tube is an Olympus nasal drainage tube ( $\alpha$-type pre-shaped, PBD21Z).

A patient with bile duct carcinoma complained of severe pharyngeal discomfort

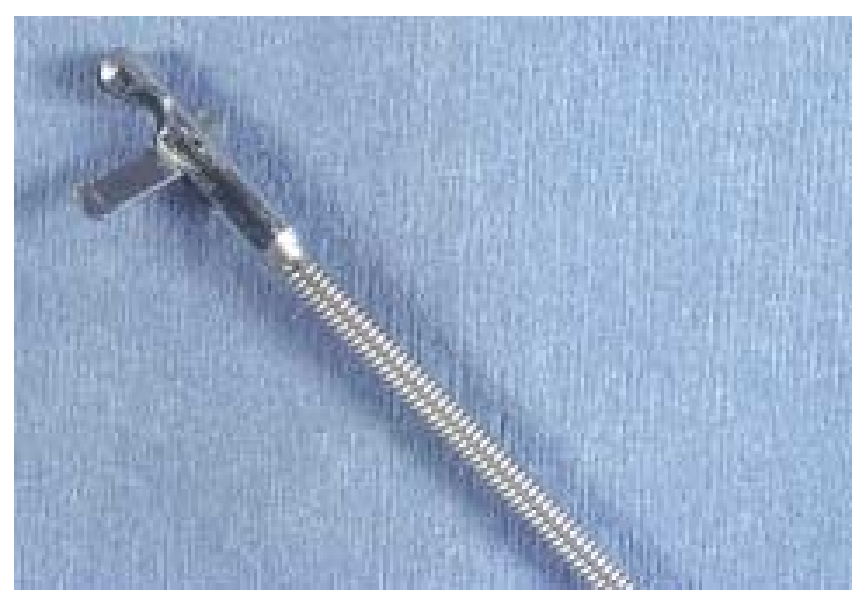

Figure 1 The scissor forceps developed by the authors were designed to hold the endoscopic nasobiliary drainage (ENBD) tube at the depressed portion so that the cutting blade was almost perpendicular to the longitudinal axis of tube when cutting

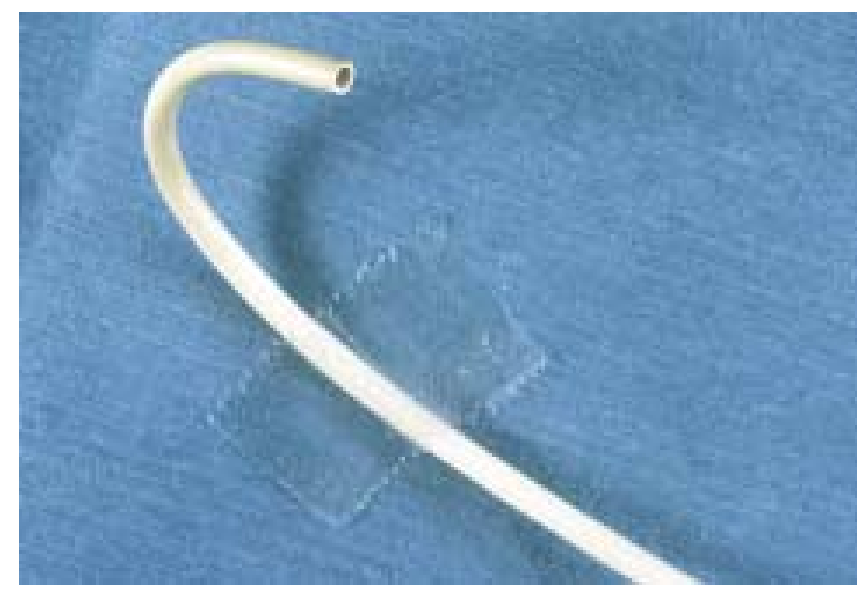

Figure 2 The cut end of the extracted tube was not sharp and no apparent deformation was seen edge of the tube, because it is unlikely that the tube will be cut at an acute angle.

Although further studies using a larger number of patients are needed, we think that our procedure is very beneficial to some patients. However, this procedure should only be used in exceptional situations, as change from a nasobiliary catheter to an internal stent would be the normal procedure of choice.

\section{N. Uchida, T. Ezaki, H. Fukuma, K. Tsutsui, H. Kobara, M. H. Bang, M. Ogawa, K. Watanabe, M. Ono,}

A. Morishita, T. Ogi, H. Kamata, T. Masaki, S. Watanabe, S. Kuriyama Third Dept. of Internal Medicine, Kagawa Medical University, Kagawa, Japan

\section{References}

${ }^{1}$ Nagai N. Study on endoscopic continuous pancreato-choledocho catheter-remaining method. Gastroenterol Endosc 1975; 17: $684-700$

\section{Corresponding Author}

\section{N. Uchida, M.D.}

Third Dept. of Internal Medicine Kagawa Medical University 1750-1 Ikenobe

Miki-cho, Kita-gun

Kagawa-ken 761-0793

Japan

Fax + 81-87-8912158

E-mail: naohito@kms.ac.jp 